\title{
PID Control of Chocolate 3D Printer Heating System
}

\author{
Long Zhuoqun, Yang Jiazhe \\ School of Electronic Engineering, Xi'an Aeronautical University, Xi’an, Shaanxi, China, 710077
}

Keywords: Chocolate 3D printing; heating; PID control

\begin{abstract}
In recent years, chocolate 3D printing technology has received extensive attention from various countries. Chocolate 3D printing has the advantages of fast molding speed, no pollution, and convenience for indoor use. It has played an important role in the development of modern manufacturing industry. This paper adopts the PID control method of the heating system of the chocolate 3D printer, which can effectively control the large-scale changes in temperature, thereby greatly improving the quality of printed work pieces.
\end{abstract}

\section{Introduction}

The study of chocolate 3D printing technology began in the 1980s when it was called rapid prototyping. The earliest research on chocolate 3D printing technology in the world was the United States 3D Systems, which introduced the first rapid prototyping machine in 1986. The chocolate 3D printing system is based on a digital model file, such as a three-dimensional CAD model. After layered and sliced, a series of two-dimensional cross-section data is obtained.

\section{Overview of Chocolate 3D Printing Technology}



Fig.1 3D Printing Output Value (Billion USD)

The data is used to control the drive system of the chocolate 3D printer to drive the nozzle to perform movement. The bondable material is obtained by stacking layer by layer to obtain a three-dimensional target entity. Currently, chocolate 3D printing technology has been widely applied to various fields such as robotics, electronic products, biomedicine, aerospace, and construction industries. Chocolate 3D printing has its unique manufacturing model that directly converts virtual digital models into products, simplifies production processes, reduces product production costs, and shortens product development cycles.3D printing is a technique for generating a three-dimensional volume by creating a continuous object layer. This technology has been applied to the production of plastics and metal products in the industry, and promotion to chocolate 
processing is the first time. The development process is no shortage of challenges. Chocolate is not easy to process, it requires precise heating and cooling cycles. Hao Liang said: "The uniqueness of this technology is that users can design and manufacture their own products. It can imitate the appearance of children's favorite toys, but also allows chocolate to look like your friend's face, can be described as unlimited creativity. This machine, users can also use different raw materials to design different products [1].3d printing output value trends as shown in fig.1.

\section{Work Procedures of Chocolate 3D Printing Technology}

By adopting different materials and molding methods, chocolate 3D printing technology continues to innovate in its implementation methods. Chocolate 3D printing technology is mainly divided into extrusion molding, granular material molding, photopolymerization molding, etc. According to the different materials and equipment, there are multiple molding methods for the above three types.

Among them, extrusion molding is the most easily realized 3D molding method, and is mainly realized by fused deposition molding (FDM) technology. The melted chocolate 3D printer controls heated nozzles through a computer and moves the X-Y two-dimensional plane according to the model-designed single-plane data. The spray head can melt the filamentary printing material sent from the extruder, and then it is extruded from the nozzle to the workbench, cooled in the air and then solidified. After printing a layer, the computer controls the height of the layer of material by the nozzle, continues to press a layer of path to complete the printing and build up, and finally realize the entire print[2].

Since the FDM technology uses industrial-grade thermoplastic materials for molding and processing, compared with other types of chocolate 3D printing, FDM molded products have good heat resistance and corrosion resistance, and have been directly obtained in mechanical parts and other products. application. The Fortus $900 \mathrm{mc}$, a super-large rapid prototyping machine developed by Stratasys in 2012, has a very high forming accuracy and a large forming size, enabling the production of product-level components. Due to the low cost of FDM implementation, currently marketed chocolate 3D printers are still mainly FDM-type devices, and the price of small-scale FDM devices has even dropped to a few thousand dollars [3].

The 3D chocolate printer is a machine that can "print" chocolate like a printed document. According to a blueprint drawn by scientists, this "3D chocolate printer" is compatible with the CAD design software used in traditional engineering computers. It knows where to put the material, line by line, and layer by layer. They believe that this new product can be used for commercial purposes in the future. As long as the popularity is successful, it will inevitably change the face of human life. The 3D chocolate printer uses a new electronic blueprint system that not only facilitates the printing of chocolate, but also helps people design different styles of chocolate. The "ink" used by the printer is all edible ingredients such as chocolate sauce, batter, cheese, etc. Once people draw a good chocolate pattern on a computer and mix it with raw materials, the electronic blueprint system will show the printer's operating procedures and complete the chocolate "build" project. This chocolate printer will greatly simplify the production process of chocolate, and at the same time, it will also help people produce more nutritious, healthy and interesting foods. This 3D chocolate printer will be available for use in different places such as homes and restaurants [4].

\section{Temperature Control System Design of Chocolate 3D Printer}

Because the extrusion head and the hot bed of the melting chocolate $3 \mathrm{D}$ printer are all in a constant temperature state, the heating resistor is used to heat it, and then the temperature is monitored by the thermistor. The microcontroller STM32F407 used in the chocolate 3D printer has a 12-bit ADC module that can acquire the voltage value of the CTCI Temp interface in real time. The thermistor connected to the interface realizes CTCI Temp interface voltage change with temperature through voltage division, and the controller ADC collects There is a good linear relationship between the voltage analog quantity and the temperature change on the thermistor, and 
the specific parameters can be calibrated by temperature calibration. The microcontroller converts the temperature into a temperature value based on the collected voltage. Then the temperature value is compared with the target temperature set by the system, and PID adjustment is performed. The CTCI Heat pin output signal drives the turn-on and turn-off of the power transistor. Control whether the heating resistance of the extruder and hot bed is heated. The micro-controller regulates the temperature of the extruder and the hot bed within a certain range through PID control to form a closed-loop temperature control system.

PID adjustment control is based on the deviation of the feedback value from the set value to perform proportional (P), integral (I) and derivative (D) operations. After calculation, an appropriate control signal is output to the execution end, prompting the feedback value to gradually approach the set value. Realize automatic control]. PID control has been widely used in engineering control. It has the advantages of simplicity, stability and convenient parameter adjustment. The PID controller adjusts the temperature by adjusting the three parameters of KP, TI and TD. e(t) is the deviation between the acquired signal and the given parameter value, which is approached to zero by PID adjustment so that the output gradually approaches the given value. At this time, the values of the three parameters are optimal. The PID operation generally needs to establish a structure data type for storing the three parameters KP, TI, TD and the accumulated value of the set value and historical error required by the PID operation.The pid formula is as follows.

$$
u(t)=K_{P}\left[e(t)+\frac{1}{T_{I}} \int_{0}^{t} e(t) d t+T_{D} \frac{d e(t)}{d t}\right]
$$

The return value of the PID operation is used to control the timer. According to the adjustment of the timer, PWM waves of different duty ratios are controlled to output, and then the working time of the heating element is controlled to obtain an almost constant temperature.

\section{PID Test Result Analysis}

In the temperature curve under PID control, the temperature rise is about 1 minute and a half, the overshoot is $5 \%$, and the steady state is reached in 3 minutes. Finally, it is stable at $180^{\circ} \mathrm{C}$ and the steady-state error is small. Without the PID control heating curve, it can be seen that the temperature fluctuation range has been around $10^{\circ} \mathrm{C}$, the overshoot is large, and the average temperature is also higher than the target temperature by $5^{\circ} \mathrm{C}$.

\section{Summary}

The temperature of PID control is obviously much more stable than that without PID control. Especially after stable operation of the extruder, the temperature fluctuation range without PID control is around $10^{\circ} \mathrm{C}$, and the average temperature is also higher than the set temperature by $5^{\circ} \mathrm{C}$. The temperature can be brought close to the set temperature under PID control. From this result analysis, the heating system of the chocolate 3D printer can accurately control the temperature through PID control, can meet the temperature requirements of the chocolate 3D printing consumables, and ensure that the PLA material is in the best melting state during the printing process. If there is no PID adjustment, the temperature of the extrusion head may fluctuate too much, and it may be because the PLA consumables are carbonized due to the high temperature, resulting in the phenomenon of blocking of the spinneret and affecting the printing effect.

\section{References}

[1] Sarah Couck, Julien Cousin-Saint-Remi, Stijn Van der Perre, Gino V. Baron, Clara Minas, Patrick Ruch, Joeri F.M. Denayer. 3D-printed SAPO-34 monoliths for gas separation[J]. Microporous and Mesoporous Materials,2018,255.

[2] Ravi K. Enneti, Kevin C. Prough, Thomas A. Wolfe, Andrew Klein, Nick Studley, Juan L. 
Trasorras. Sintering of WC-12\%Co processed by binder jet 3D printing (BJ3DP) technology[J]. International Journal of Refractory Metals and Hard Materials,2018,71.

[3] Henry Oliver T. Ware, Adam C. Farsheed, Banu Akar, Chongwen Duan, Xiangfan Chen, Guillermo Ameer, Cheng Sun. High-speed on-demand 3D printed bioresorbable vascular scaffolds[J]. Materials Today Chemistry,2018,7.

[4] Luana Persano, Francesco Cardarelli, Arkadii Arinstein, Sureeporn Uttiya, Eyal Zussman, Dario Pisignano, Andrea Camposeo. 3D printing of optical materials: an investigation of the microscopic properties[P]. OPTO,2018. 